\title{
On Agulhas eddies and Rossby waves travelling by forcing effects
}

\author{
V. G. Gnevyshev ${ }^{1}$, A. A. Malysheva ${ }^{2}$, T. V. Belonenko ${ }^{2}$, and A. V. Koldunov²
}

Received 12 April 2021; accepted 24 April 2021; published 1 October 2021.

This study analyzes tracks of Agulhas eddies in the South Atlantic. The research is based on the product "Mesoscale Eddies in Altimeter Observations of SSH". The method of automatic eddy identification detects 54,496 eddies for 1993-2017: 28,018 cyclones and 26,478 anticyclones. We show that only anticyclones are long-lived eddies in the region. We analyze 15 tracks of the anticyclones, that have more than a 2.5-year lifetime period. We find that these eddies, crossing the South Atlantic, have an almost rectilinear motion to the northwest. Their parameters (amplitude, radius, orbital velocity, and speed of drift) change during their life, however, we do not find either an explicit dependence of the displacement on topography or attenuation of eddies over time. The zonal displacement and, accordingly, the zonal component of the displacement velocity dominate, however, in some parts of the track, the vortex displacement to the equator is comparable to their zonal displacement. We hypothesize that the main problem of the inapplicability of most analytical models for interpretation of the meridional displacement is ignoring the initial values when generating eddies. We propose an alternative theory where the evolution of the initial stage of the generation of Agulhas eddies is consistent with the directional angular emission of long Rossby waves by non-zonal currents. Based on this theory, we analyze the tracks of the long-lived eddies. The proposed interpretation allows us to explain many of the observed effects but does not reject other possible scenarios for other regions of the World Ocean. KEYWORDS: Altimetry; sea level anomalies; Agulhas eddies; mesoscale eddy; anticyclones; track; Rossby wave; wave radiation; meridional displacement; automatic method of identification.

Citation: Gnevyshev, V. G., A. A. Malysheva, T. V. Belonenko, and A. V. Koldunov (2021), On Agulhas eddies and Rossby waves travelling by forcing effects, Russ. J. Earth. Sci., 21, ES5003, doi:10.2205/2021ES000773.

\section{Introduction}

Agulhas eddies are formed as a result of the instability of the Agulhas Current near the southern tip of Africa. All are more than $100 \mathrm{~km}$ in size, extend deep in the water up to $1000 \mathrm{~m}$, and drift westward from their respective formation sites. Ag-

\footnotetext{
${ }^{1}$ Shirshov Institute of Oceanology RAS, Moscow, Russia

${ }^{2}$ Saint Petersburg State University, St. Petersburg, Russia
}

Copyright 2021 by the Geophysical Center RAS. http://rjes.wdcb.ru/doi/2021ES000773-res.html ulhas eddies crossing the South Atlantic may reach the Brazil Current region. Guerra et al. 2018 provided a great overview of this phenomenon. The influence of Agulhas leakage on the main circulation in the Atlantic Ocean occurs in two ways: through the radiation of planetary waves and advection [Biastoch et al., 2008]. Entering the region of the Cape Agulhas, warmer and more salty waters compared to the environment, deflect and deepen isohaline and isotherms in the thermocline [Giulivi and Gordon, 2006] and create in isopycnic medium heterogeneities which can radiate Rossby waves spreading slowly across the South Atlantic [Biastoch et al.,2008; Gnevyshev et al.,2019; Maly- 
sheva et al., 2020a. Agulhas eddies generated by barotropic and baroclinic instability of the Agulhas Current [Gordon and Haxby, 1990, Guerra et al., 2018, Malysheva et al., 2020a, 2020b cross the South Atlantic feeding the surface limb of the Atlantic Meridional Overturning Circulation with warm and salty waters original from the Indian Ocean. Agulhas eddies keep their thermohaline characteristics while moving to the west and transferring heat and salt from the Indian to the Atlantic Ocean. Using a combination of altimetry and Argo observations, Malysheva et al. 2020a. $2020 \mathrm{~b}$ estimated the mean Agulhas leakage transport by one mesoscale eddy is $8.5 \mathrm{~Sv}$. The heat and salt transports by the individual Agulhas leakage eddy are $2.25 \times 10^{9} \mathrm{~W}$ and $5.36 \times 10^{5} \mathrm{~kg} \mathrm{~s}^{-1}$, respectively. Annual heat and salt transports by eddies are $2.03 \times 10^{15} \mathrm{~J}$ and $4.83 \times 10^{11} \mathrm{~kg}$, respectively [Malysheva et al., 2020a, 2020b], see also [Belonenko et al., 2020].

Analysis of altimetry maps of SLA (Sea Level Anomalies) shows steady westward propagation of mesoscale eddies in the World Ocean, which is explained by the existence of planetary Rossby waves. However, several researchers noticed at the same time that there is a significant meridional displacement in eddy transfer herewith cyclones tend to move poleward and anticyclones equatorward [see e.g. [Cushman-Roisin, 1994 Early et al., 2011, Reznik, 2010, Reznik and Tsybaneva, 1994. Chelton et al. [2011] and Morrow et al. 2004] believe that this displacement can be explained by a modification of the potential vorticity gradient by vertically sheared mean flow. They suggest that this is an effect of the spherical geometry of the Earth on the large eddies, which cause equatorward bias because of their large latitudinal size. Another explanation is the large nonlinearity of eddies. Kamenkovich et al. 1996 notice that the Agulhas eddies are nonlinear with Rossby number $R_{0}<0.1$. Due to their nonlinearity, eddies have a meridional component to their translation velocity, thereby in the Southern Hemisphere, anticyclonic eddies tend to move to the northwest contrary to cyclones, which move to the southwest. Despite its importance, the problem of the influence of nonlinearity on moving eddies has not been sufficiently addressed. Besides, any acceptable verification of the phenomena of observations for the theories does not exist nowadays.
The main purpose of this paper is to compare the existing theoretical approaches to the phenomenon. We are based on the altimetry maps of the South Atlantic. Our main interest is the meridional bias of Agulhas eddies advection.

In our study, only long-lived Agulhas eddies are considered, the lifespan of which exceeds 2.5 years. It turned out that only anticyclones satisfy this criterion since all formed cyclones dissipate within the first year after their generation, that is, cyclones are much less viable than anticyclones. This fact is explained in the study of Nezlin 1986 in terms of cyclone-anticyclone asymmetry. He explains more sustainability of anticyclones in comparison with cyclones by the fact that the speed rotor of a cyclone is parallel to the velocity vector of the Earth's rotation, while that of an anticyclone is antiparallel. This means that in the equation for the conservation of a potential vortex, scalar nonlinearity can balance the dispersion only in the case of an anticyclone. For a cyclone, dispersion and scalar nonlinearity have the same signs and, therefore, cannot be mutually compensated. Thus, the observed cyclone-anticyclone asymmetry is a direct consequence of scalar nonlinearity and a dispersionnonlinear property of Rossby vortices, which is a very fundamental law.

The presence of cyclone-anticyclonic asymmetry in the conservation laws largely determines the possibility (or impossibility) of the formation of solitary vortices of different polarities. This means that if there is a "scalar" Rossby soliton (a solitary wave in which dispersive spreading is compensated by twisting caused by scalar nonlinearity), then it can only be an anticyclone, that is, only an elevation soliton, like the classical Russell soliton or the quasi-permanent anticyclonic Lofoten vortex in the Norwegian Sea [Travkin and Belonenko, 2019. Nezlin [1986 also points out that almost all large longlived eddies on major planets, like Jupiter's Great Red Spot, apparently are anticyclones, that is, it indicates cyclone-anticyclonic asymmetry. According to Nezlin [1986, the wave approach is more informative for the study of eddies in the ocean, that is what makes it possible to simply explain such a fundamental phenomenon as the cycloneanticyclonic asymmetry.

Thus, according to Nezlin, anticyclones in the ocean are Rossby vortices (Rossby solitons), and its properties (dimensions, direction, and drift ve- 
locity) are well predicted and described based on wave concepts, according to which the Rossby vortex is the result of a mutual balancing of dispersion and nonlinearity. Consequently, a Rossby vortex (soliton) is an object exhibiting an explicit dualism, and therefore the frequently asked question: "Is this a vortex or a wave?", it is not quite correct. Such an object can equally well be called either a wave solitary (i.e. non-spreading) vortex or a vortex soliton, depending on which of its properties are considered.

Thus, in this paper, we analyze only anticyclones - long-lived eddies of the Agulhas current and meridional displacement of them during their moving in the South Atlantic. According to $\mathrm{Ne}$ zlin 1986, we can also call these anticyclones the Rossby vortices (or Rossby solitons). Specifically, we aim to answer the following questions: (i) Why do anticyclones move not only westward but towards the equator? (ii) What characteristics do they have? (iii) How does the bottom topography affect their movement? (iv) What are the zonal and meridional displacements of these eddies? (v) What existing theories can better match these estimates? To answer these questions, we analyze satellite altimetry data and specifically the altimeter products "Mesoscale Eddies in Altimeter Observations of SSH" [Chelton et al., 2011]. Besides, we discuss several theories that also try to explain the northwest displacement of moving eddies. Finally, we suggest our explanation of the phenomena.

\section{Data}

The altimeter products were produced
by SSALTO/DUACS and distributed
by AVISO, with support from CNES
(http://www.aviso.oceanobs.com/duacs/).
The product is available on AVISO (https://www.aviso.altimetry.fr). The specially developed eddy identification algorithm based on the analysis of altimetry images is used for creating this data. The method of eddy identification and tracking using Sea Surface Height (SSH) described in Chelton et al. 2011 is modified in a manner inspired by Williams et al. 2011.

The essence of the original algorithm was as follows. A single mesoscale eddy, identified us- ing an automatic algorithm, was identified as a linked bulk of pixels that satisfy a certain number of criteria, such as the presence of a local maximum (minimum) in the SSH for an anticyclonic (cyclonic) eddy, the consistency of the anomalies values within the contour of this cluster with its extremum sign. The maximum eddy size was limited to 1000 pixels, the minimum was 8 pixels, and the minimum eddy amplitude was $1 \mathrm{~cm}(1 \mathrm{~cm}$ discretization of the step size). A more detailed description of these criteria is provided in Chelton et al. 2011. The data contains several parameters of interest: coordinates $\left(x_{c}, y_{c}\right)$ of the eddy center based on SSH (Sea Surface Height), amplitude, which is defined as the difference between the SSH value of extremum and the average $\mathrm{SSH}$ value at the eddy boundary, eddy radius, which is calculated through the area closed isolines characterizing the eddy, orbital velocity. Using the coordinates of the center and the date, we also calculated the speed of the eddy.

We also use AVISO geostrophic velocities from 01 January 1993 to 31 December 2017, provided by CMEMS and distributed by E.U. Copernicus Marine Service.

The bottom topography data of the study area was taken from the Global Relief Model of the Earth's surface ETOPO1 (https://www.ngdc.noaa. gov). The model is implemented on a grid with a spatial resolution of $1 / 60^{\circ}$. ETOPO1 is vertically referenced to sea level and horizontally referenced to the World Geodetic System 1984 (WGS 84). The Global Relief Model is a synthesis of various global and regional datasets.

\section{Results}

\subsection{Analysis of Agulhas Eddies Tracking}

The study area is shown in Figure 1, which reflects the main topographic formations. The velocities of currents in the central part of the region are low and directed mainly to the northwest. An eddy identification algorithm identifies 54,496 unique mesoscale eddies in the area for 1993-2017, of which 28,018 are cyclones and 26,478 are anticyclones. The total number of eddies identified in every bin $3.0^{\circ} \times 3.0^{\circ}$ is shown in Figure 2 a. It can be seen that a lot of eddies have a topographic na- 


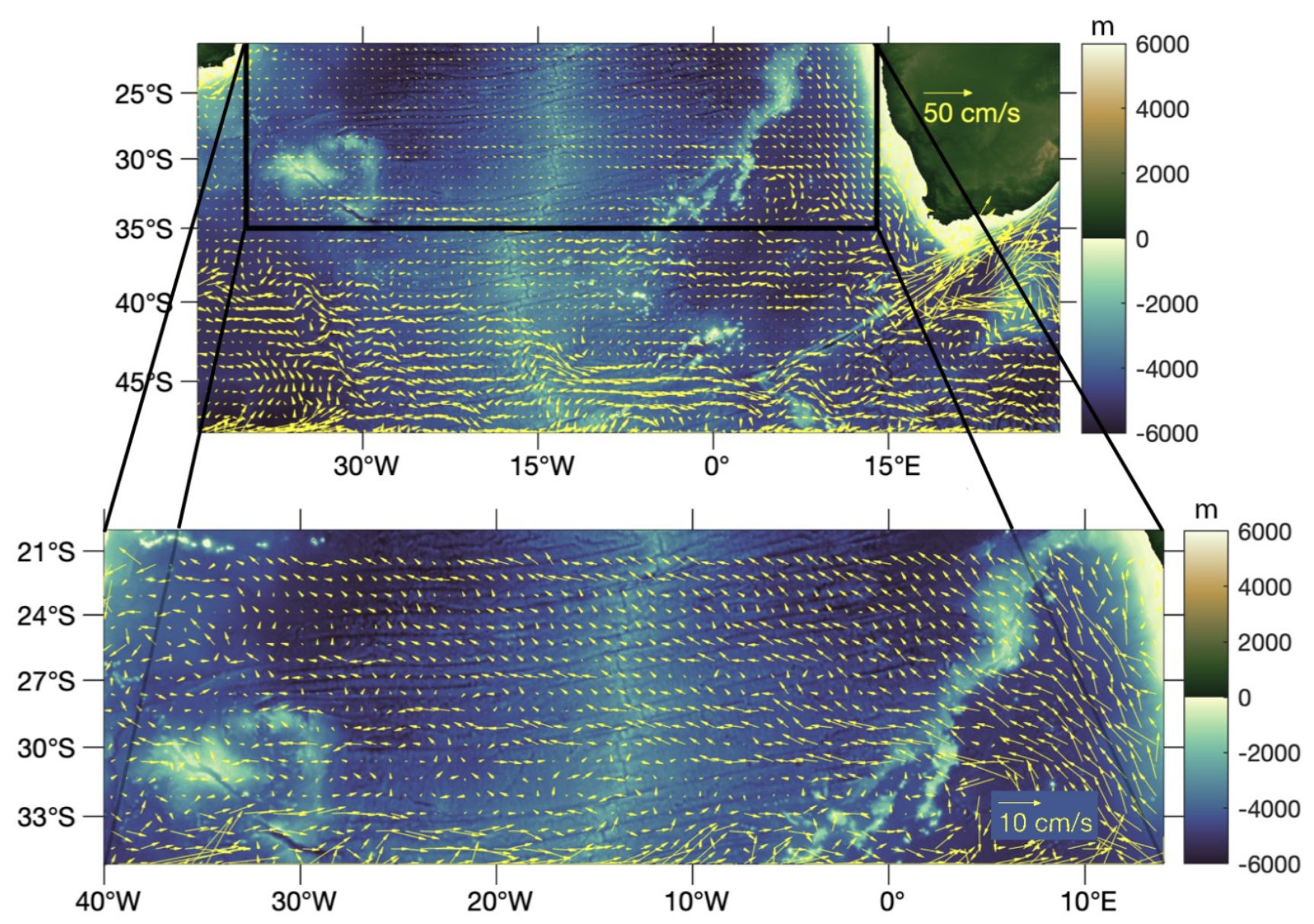

Figure 1. The study area (the bottom panel). The color scale shows the depth (m). Arrows show the vectors of current velocities to the west of $30^{\circ} \mathrm{E}$, calculated from altimetry data (averaging 1993-2017).

ture and are confined to the main topographic formations. It is important to note that most eddies have a short lifespan and quickly dissipate.

In our study, only long-lived eddies were selected for research. These eddies move in the South Atlantic and go to extraordinary distances. Figure 2b shows the tracks of 15 Agulhas eddied that we selected for analysis. We note that although the total number of cyclones in the "Mesoscale Eddy Trajectory Atlas Product" exceeds the number of anticyclones, it turned out that only anticyclones are long-lived. The anticyclones in Figure $2 \mathrm{~b}$ have a lifespan of more than 2.5 years. In the further presentation, the anticyclone track numbers correspond to their numbers in the product used "Mesoscale Eddy Trajectory Atlas Product" (see Supporting Material). We divided each track of the eddy into $3-6$ parts according to its direction changes relative to latitude when the eddy moved (Table 1).

We note that anticyclones mainly move to the northwest, thereby we qualitatively confirm that different types of anticyclone trajectories are not radically different from each other, especially at large times, although some eddies have a southern velocity component. On each part of the track, we consider the vortex characteristics to be constant. Table 1 shows the characteristics of these eddies on parts of the track. It can be seen that the average radius of eddies varies from 47 to $162 \mathrm{~km}$, the amplitude is from 3 to $18 \mathrm{~cm}$, the orbital velocity is from 55 to $171 \mathrm{~cm}$, the velocity of movement is from 1.2 to $9.6 \mathrm{~cm} \mathrm{~s}^{-1}$, the total lifetime of vortices is from 900 to 1198 days. Figure 2b and Table 1 show that all considered eddies have a meridional displacement. The zonal displacement exceeds the 
Table 1. Parameters of Long-Lived Mesoscale Eddies in the South Atlantic

\begin{tabular}{|c|c|c|c|c|c|c|c|c|c|}
\hline Track No & $\begin{array}{l}\text { Mean } \\
\text { radius } \\
R, \mathrm{~km}\end{array}$ & $\begin{array}{c}\text { Mean } \\
\text { amplitude } \\
A, \mathrm{~cm}\end{array}$ & $\begin{array}{c}\text { Orbital } \\
\text { velocity } \\
V_{0}, \mathrm{~cm} \mathrm{~s}^{-1}\end{array}$ & $\begin{array}{c}\text { Speed } \\
\text { of drift } \\
V_{d}, \mathrm{~cm} \mathrm{~s}^{-1}\end{array}$ & $\begin{array}{c}\text { zonal } 1 \\
L_{x}, \mathrm{~km}\end{array}$ & $\begin{array}{r}\text { Displa } \\
L_{x}, \text { meridi } \\
L_{y}, \mathrm{~km}\end{array}$ & $\begin{array}{l}\text { cement: } \\
\text { nal } L_{y} ; \mathrm{d} \\
T \text {, days }\end{array}$ & $\begin{array}{c}\text { stance } S \\
S, \mathrm{~km}\end{array}$ & $\begin{array}{c}\text { Total } \\
\text { lifetime, } \\
\text { days }\end{array}$ \\
\hline 139147.1 & 86 & 7 & 74 & 5 & 558 & 555 & 220 & 795 & 1198 \\
\hline 139147.2 & 87 & 5 & 70 & 4 & 1173 & 111 & 390 & 1377 & \\
\hline 139147.3 & 95 & 3 & 65 & 4.3 & 1173 & 277 & 293 & 1247 & \\
\hline 139147.4 & 81 & 6 & 65 & 3.9 & 1017 & 218 & 280 & 1248 & \\
\hline 139147.5 & 71 & 3 & 55 & 1.2 & 207 & 118 & 15 & 69 & \\
\hline 64204.1 & 89 & 5 & 65 & 3.3 & 540 & 331 & 190 & 572 & 1096 \\
\hline 64204.2 & 122 & 3 & 113 & 5.3 & 1680 & 442 & 380 & 1812 & \\
\hline 64204.3 & 60 & 3 & 75 & 5.5 & 1523 & 192 & 330 & 1612 & \\
\hline 64204.4 & 78 & 3 & 67 & 6.5 & 1108 & 332 & 196 & 1139 & \\
\hline 84755.1 & 72 & 4 & 61 & 7.5 & 766 & 221 & 115 & 770 & 1088 \\
\hline 84755.2 & 86 & 6 & 73 & 4.5 & 466 & -221 & 130 & 520 & \\
\hline 84755.3 & 74 & 4 & 75 & 8.6 & 421 & 9.5 & 55 & 421 & \\
\hline 84755.4 & 73 & 4 & 62 & 9.3 & 933 & 321 & 200 & 966 & \\
\hline 84755.5 & 77 & 3 & 81 & 4.5 & 1906 & 221 & 480 & 1937 & \\
\hline 84755.6 & 74 & 4 & 87 & 4.6 & 584 & -9.5 & 108 & 389 & \\
\hline 138285.1 & 60 & 10 & 64 & 5.6 & 280 & 277 & 70 & 349 & 1130 \\
\hline 138285.2 & 60 & 8 & 91 & 2 & 187 & -111 & 130 & 216 & \\
\hline 138285.3 & 80 & 4 & 65 & 3 & 373 & 332 & 180 & 438 & \\
\hline 138285.4 & 77 & 4 & 85 & 3.8 & 1264 & 443 & 380 & 1288 & \\
\hline 138285.5 & 76 & 4 & 84 & 5.3 & 1178 & 442 & 270 & 1278 & \\
\hline 138285.6 & 61 & 3 & 82 & 7.2 & 332 & 120 & 100 & 648 & \\
\hline 78168.1 & 60 & 18 & 115 & 4.3 & 685 & 591 & 210 & 804 & 1057 \\
\hline 78168.2 & 79 & 11 & 69 & 6 & 3606 & 664 & 690 & 3773 & \\
\hline 78168.3 & 70 & 6 & 75 & 7.2 & 600 & -120 & 80 & 515 & \\
\hline 78168.4 & 67 & 6 & 73 & 8.7 & 199 & -563 & 77 & 596 & \\
\hline 61092.1 & 68 & 6 & 76 & 4.5 & 721 & 655 & 250 & 994 & 1045 \\
\hline 61092.2 & 90 & 4 & 77 & 2.7 & 295 & 212 & 150 & 361 & \\
\hline 61092.3 & 78 & 4 & 91 & 5 & 2900 & 212 & 645 & 2919 & \\
\hline 56389.1 & 74 & 10 & 69 & 3.3 & 1124 & 221 & 390 & 1158 & 954 \\
\hline 56389.2 & 72 & 7 & 69 & 4.7 & 1400 & 220 & 340 & 1446 & \\
\hline 56389.3 & 65 & 4 & 68 & 5.2 & 212 & 111 & 234 & 1080 & \\
\hline 44929.1 & 88 & 9 & 71 & 5.5 & 2230 & 323 & 460 & 2243 & 948 \\
\hline 44929.2 & 85 & 8 & 68 & 6 & 2332 & 212 & 440 & 2364 & \\
\hline 44929.3 & 75 & 7 & 58 & 3.6 & 97 & -100 & 48 & 155 & \\
\hline 83056.1 & 65 & 3 & 85 & 9.6 & 267 & -120 & 50 & 430 & 936 \\
\hline 83056.2 & 78 & 4 & 86 & 6.3 & 1353 & 656 & 260 & 1463 & \\
\hline 83056.3 & 74 & 5 & 73 & 5.2 & 2900 & 655 & 626 & 2915 & \\
\hline 62705.1 & 81 & 7 & 79 & 2.7 & 460 & 110 & 200 & 481 & 922 \\
\hline 62705.2 & 94 & 3 & 71 & 5.4 & 462 & 101 & 100 & 484 & \\
\hline 62705.3 & 162 & 7 & 55 & 4.7 & 350 & -9.5 & 90 & 377 & \\
\hline 62705.4 & 95 & 4 & 67 & 3.1 & 600 & 212 & 220 & 617 & \\
\hline 62705.5 & 86 & 8 & 113 & 3.7 & 663 & 101 & 210 & 693 & \\
\hline 62705.6 & 70 & 12 & 57 & 3.3 & 275 & -12 & 102 & 299 & \\
\hline
\end{tabular}


Table 1. Parameters of Long-Lived Mesoscale Eddies in the South Atlantic (Continued)

\begin{tabular}{|c|c|c|c|c|c|c|c|c|c|}
\hline Track No & $\begin{array}{l}\text { Mean } \\
\text { radius } \\
R, \mathrm{~km}\end{array}$ & $\begin{array}{c}\text { Mean } \\
\text { amplitude } \\
A, \mathrm{~cm}\end{array}$ & $\begin{array}{c}\text { Orbital } \\
\text { velocity } \\
V_{0}, \mathrm{~cm} \mathrm{~s}^{-1}\end{array}$ & $\begin{array}{c}\text { Speed } \\
\text { of drift } \\
V_{d}, \mathrm{~cm} \mathrm{~s}^{-1}\end{array}$ & $\begin{array}{c}\text { zonal } \\
L_{x}, \mathrm{~km}\end{array}$ & $\begin{array}{r}\text { Displa } \\
L_{x}, \text { meridi } \\
L_{y}, \mathrm{~km}\end{array}$ & $\begin{array}{l}\text { cement: } \\
\text { nal } L_{y} ; \mathrm{d} \\
T \text {, days }\end{array}$ & $\begin{array}{l}\text { stance } S \\
S, \mathrm{~km}\end{array}$ & $\begin{array}{c}\text { Total } \\
\text { lifetime, } \\
\text { days }\end{array}$ \\
\hline 116552.1 & 71 & 4 & 64 & 3 & 115 & -102 & 49 & 133 & 922 \\
\hline 116552.2 & 76 & 11 & 88 & 6.4 & 280 & 101 & 51 & 292 & \\
\hline 116552.3 & 59 & 11 & 72 & 5 & 483 & -100 & 110 & 492 & \\
\hline 116552.4 & 75 & 4 & 60 & 3 & 223 & -98 & 90 & 240 & \\
\hline 116552.5 & 74 & 4 & 56 & 5.2 & 2800 & 664 & 622 & 2870 & \\
\hline 293011.1 & 61 & 5 & 90 & 4.5 & 781 & -236 & 200 & 794 & 905 \\
\hline 293011.2 & 88 & 4 & 62 & 5.6 & 1533 & 12 & 305 & 1533 & \\
\hline 293011.3 & 56 & 4 & 66 & 4.7 & 1039 & -12 & 250 & 1058 & \\
\hline 293011.4 & 88 & 3 & 52 & 2.5 & 100 & -9.5 & 50 & 111 & \\
\hline 293011.5 & 66 & 3 & 75 & 1.5 & 92 & 9.5 & 100 & 137 & \\
\hline 102254.1 & 57 & 7 & 94 & 7.1 & 369 & 323 & 60 & 381 & 942 \\
\hline 102254.2 & 51 & 8 & 126 & 5.2 & 140 & -9.5 & 40 & 185 & \\
\hline 102254.3 & 62 & 9 & 78 & 3.2 & 500 & 453 & 220 & 641 & \\
\hline 102254.4 & 115 & 5 & 86 & 4.8 & 2590 & 533 & 612 & 2586 & \\
\hline 13155.1 & 71 & 11 & 63 & 4.5 & 1546 & 221 & 380 & 1545 & 902 \\
\hline 13155.2 & 58 & 6 & 69 & 5.5 & 1619 & 221 & 340 & 1651 & \\
\hline 13155.3 & 47 & 6 & 63 & 4.3 & 486 & 110 & 130 & 500 & \\
\hline 13155.4 & 58 & 5 & 67 & 4.7 & 200 & -101 & 52 & 218 & \\
\hline 78132.1 & 70 & 6 & 98 & 3.1 & 265 & 9 & 105 & 267 & 900 \\
\hline 78132.2 & 83 & 9 & 108 & 3.7 & 1 & 341 & 115 & 346 & \\
\hline 78132.3 & 83 & 8 & 139 & 4.5 & 1902 & 895 & 515 & 2036 & \\
\hline 78132.4 & 80 & 8 & 171 & 4.8 & 604 & 230 & 165 & 642 & \\
\hline
\end{tabular}

meridional one in all cases, and, it is significant. Note that the values of displacement can be comparable in certain parts of the tracks.

\subsection{Role of Bottom Topography}

The influence of the relief, and especially of the ridges, on the dynamics and spatial distribution of mesoscale eddies should be particularly noted [Gnevyshev et al., 2021. Figure 1b shows that the bottom topography affects the propagation of eddies. Moreover, eddies are often formed on the surface roughness of the relief under the influence of many factors. All analyzed anticyclone tracks originate from the southwestern coast of Africa (Figure $2 \mathrm{~b}$ ) and are mainly directed to the northwest. It is easy to see that the observed anomalies have strong coherence with the passage of topographic features, which indirectly confirms the influence of topography on the speed and direction of the eddy propagation. Leonov 1995 shows that the Agulhas eddies slow down their movement during the crossing of the ridge, and can also be stopped by the ridge or even destroyed [Byrne et al., 1995. According to Korotayev et al. [1997, anticyclones are reluctant to cross the ridges. They make a gentle ascent due to the reserve of translational momentum. The northern component of the speed decreases when crossing the ridges (see Table 1. tracks 139147, 44929, 61092, 83056, 62705, 116552, 293011, 102254). Korotaev et al. 1997 say: "Particularly, a cyclonic vortex tends to drift to the top of a hill or a ridge. An anticyclonic vortex, on the contrary, slides to the bottom of a valley or a trough". This seems to be consistent with Leonov 1995 and Benilov 2005 as well. 

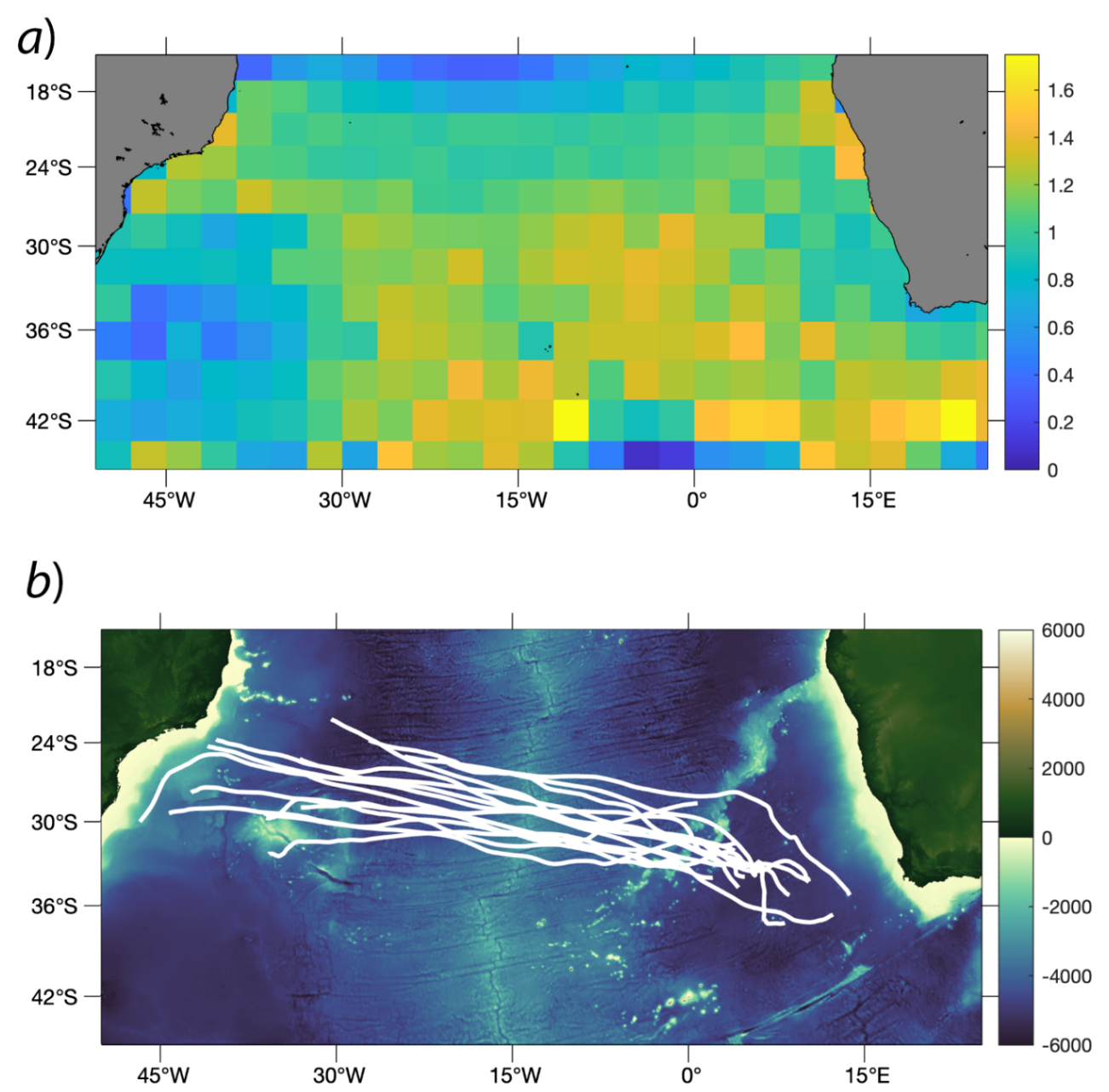

Figure 2. Average (1993-2017) daily number of mesoscale eddies identified with the automatic method by Chelton et al. 2011 in every bin $3.0^{\circ} \times 3.0^{\circ}$ (a); tracks (shown by white) of long-lived anticyclones crossing the South Atlantic (b).

Leonov 1995 also notes that the average velocities of the eddies crossing the ridge are a little dependent on the initial vertical structure of the eddies, however, there is the following regularity: the greater the advection velocity of a vortex, the greater its meridional displacement per unit of time. This is also confirmed by our eddies, see tracks: 139147, 78168, 83056, 62705, 102254, 78132.

Among the considered anticyclones, there are many examples of when eddies cross large landforms. Under the influence of topography, especially when crossing ridges, practically rectilinear tracks change azimuth (Figure 3 ) and change the angle then propagate again almost rectilinearly (see, pls, Suppl. Mat.) Note that the character of crossing the ridges by eddies is somewhat simi- lar to the refraction of a ray of light when passing through a prism. Before passing the ridge and after passing the ridge, the trajectory is quasi-linear. The influence of the ridge changes the direction of movement. There is a kind of refraction of the eddy trajectory on the ridge.

\subsection{Verification of Altimetry Observations with Theoretical Models}

3.3.1. Analysis of existing appropriate models concerning to meridional displacement of eddies. Based on a qualitative analysis of the long-term evolution of isolated eddies, the eddy amplitudes and radii, it is impossible to confirm the conclusion about systematic decrease 


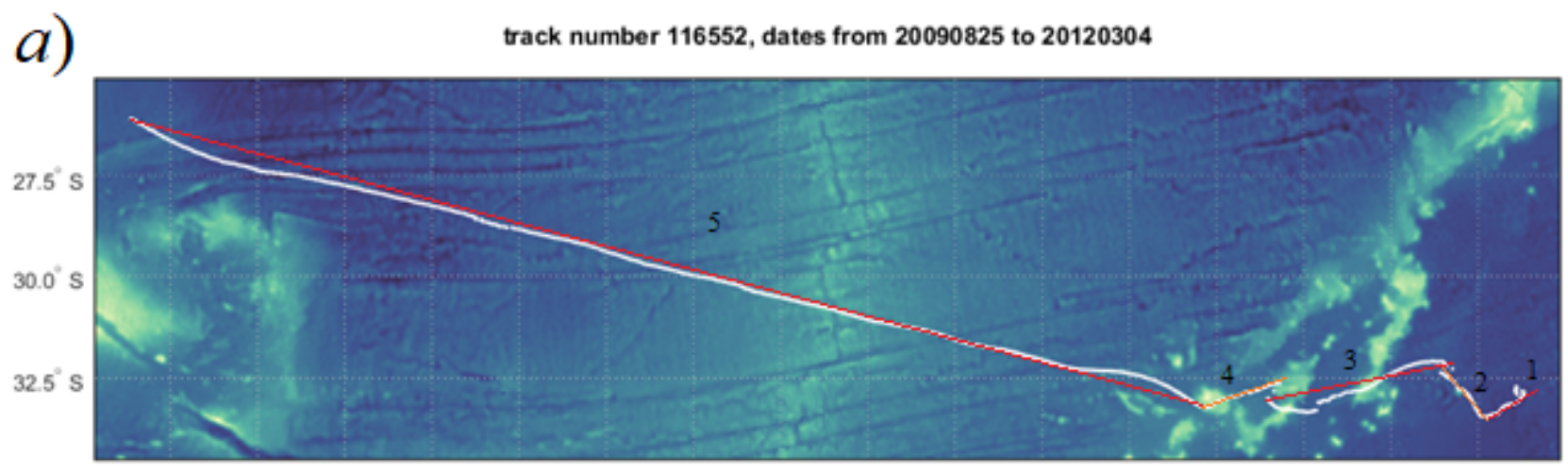

$32.5^{\circ}$ W $30.0^{\circ}$ W $27.5^{\circ}$ W $25.0^{\circ}$ W $22.5^{\circ}$ W $20.0^{\circ}$ W $17.5^{\circ}$ W $15.0^{\circ}$ W $12.5^{\circ}$ W $10.0^{\circ}$ W $7.5^{\circ}$ W $5.0^{\circ}$ W $2.5^{\circ}$ W $0.0^{\circ} \quad 2.5^{\circ} \mathrm{E} \quad 5.0^{\circ} \mathrm{E}$

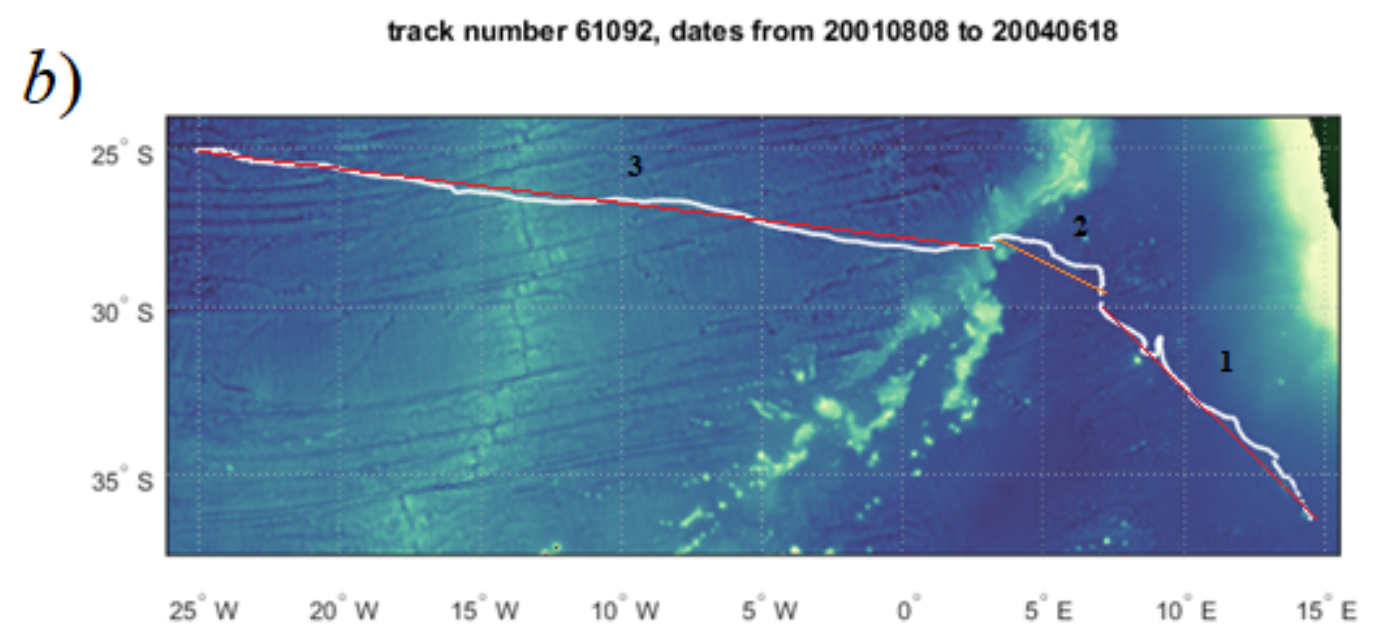

Figure 3. Tracks (the white lines) of the anticyclones crossing the South Atlantic: a) the track 116552 identified from 25.08.2009 to 04.03.2012; b) the track 61092 identified from 08.08.2001 to 18.06.2004. The red lines divide the tracks into some parts (shown in numbers).

energy with time (at large times). This tendency is described by Korotaev and Fedotov 1994 and Korotaev 1997, as well as by Reznik and Dewar 1994 , which explain this decrease by the radiation of Rossby waves by eddies. However, the movement of eddies can be due to the absorption of Rossby waves and the fact that there is a critical value of the eddy amplitude, below which there is no radiation of Rossby waves [Korotaev et al., 1997. On the contrary, a seemingly inexplicable short-term increase in the eddy scale can occur. This does not appear that it is related to topographic reasons (track 116552, see Figure 4). Probably the enhancement is due to the baroclinic factor, which is not usually taken into account by the available analytical models due to the complexity of the problem statement. This is also supported by the findings of Early et al. 2011 that, possibly, the factor associated with the radiation of Rossby waves by a vortex may be strongly overestimated at long times when entering the quasi-stable state. Guerra et al. 2018 used the automatic identification method to detect mesoscale eddies in the South Atlantic. They also indicated the existence of an "eddy corridor". They point out that the ridges constitute obstacles to an eddy drift in the Atlantic, as they usually show a deflection of trajectory and reduction of drift speed, while they manage to pass through the 

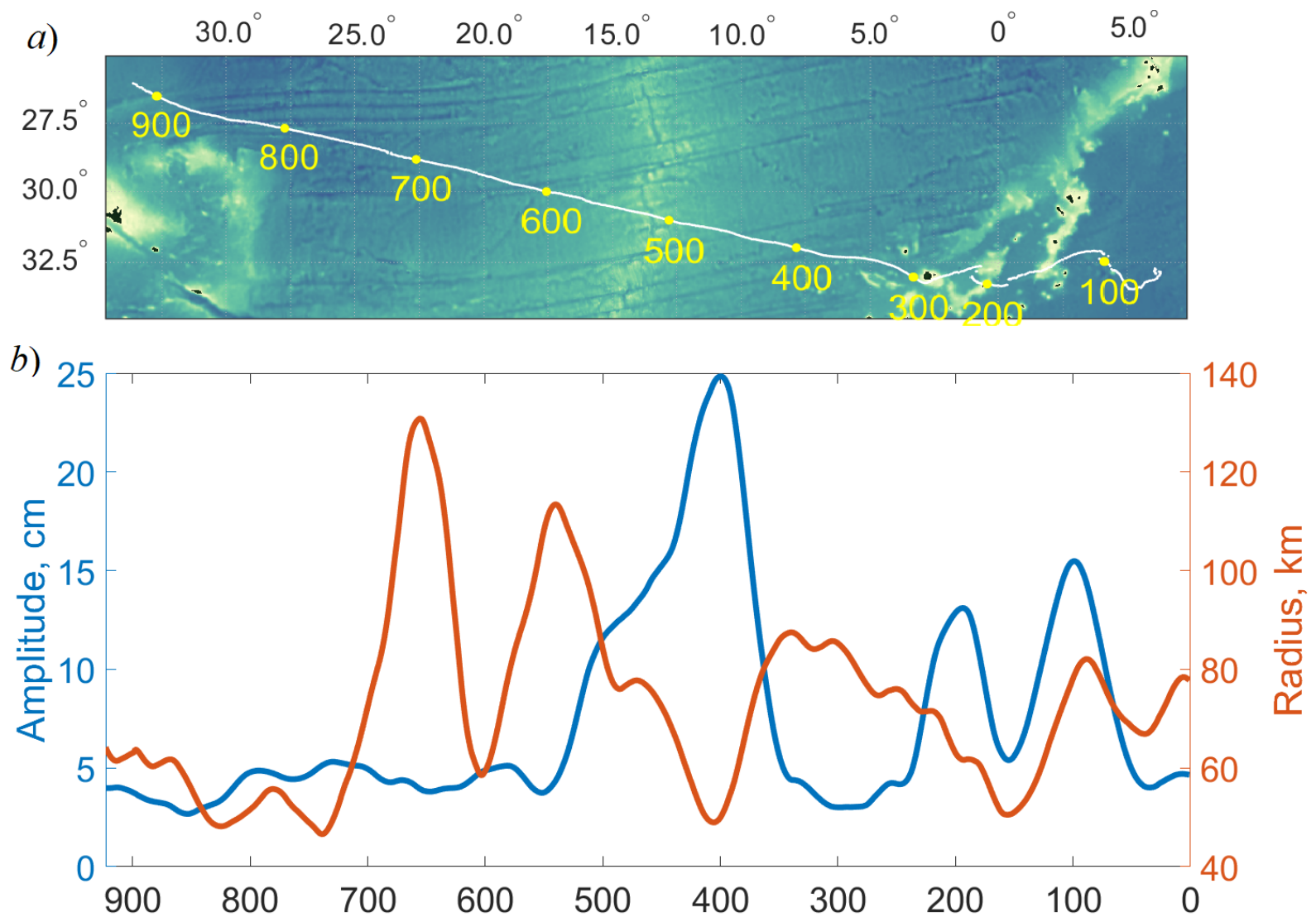

c)

Days

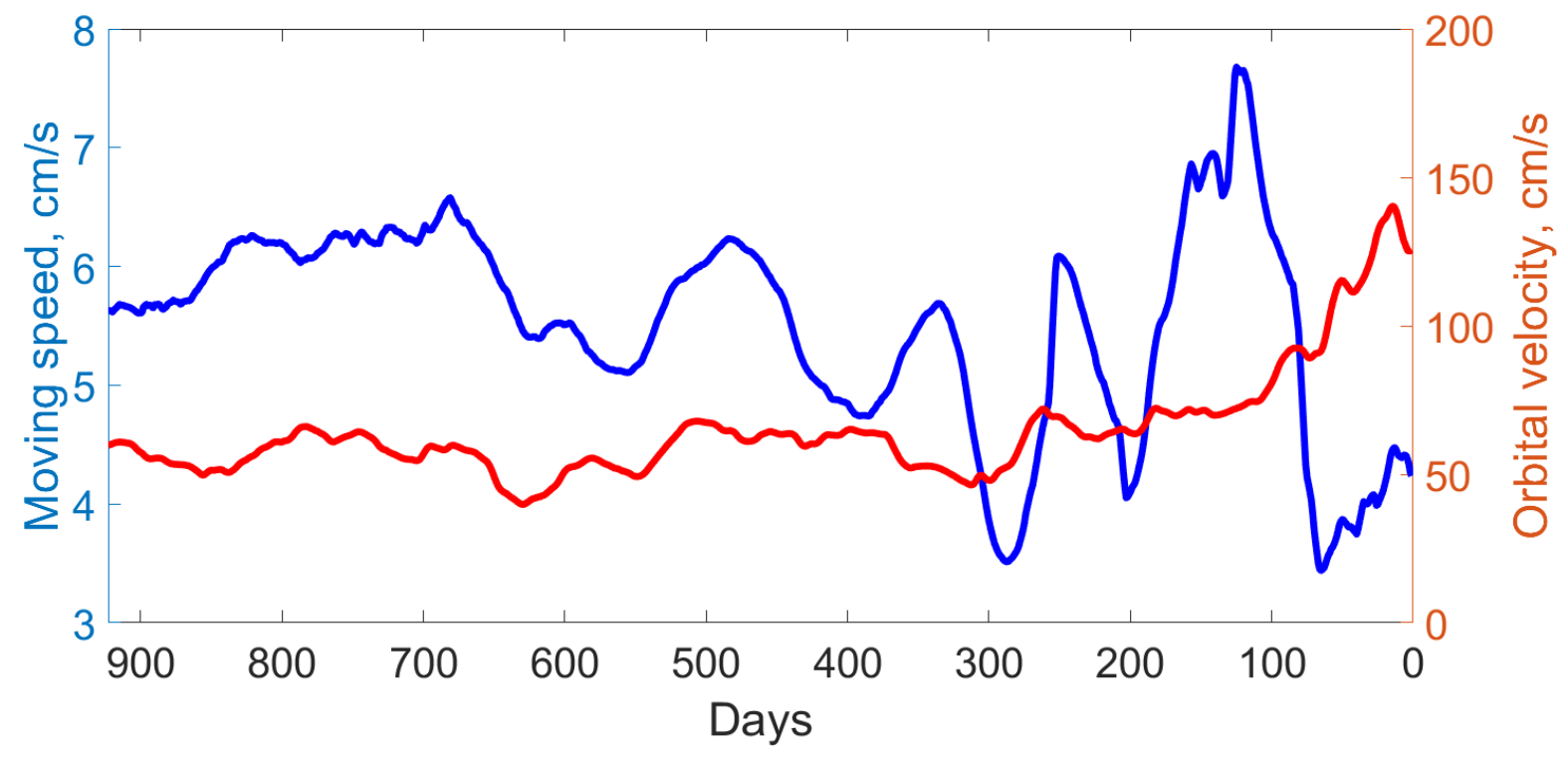

Figure 4. Track 116552: the numbers correspond to the day of the eddy existence from its generation (a); Time variability of amplitude and radius of the eddy (b), moving speed and orbital velocity (c). 


\section{track number 83056, dates from 20041016 to 20070510}

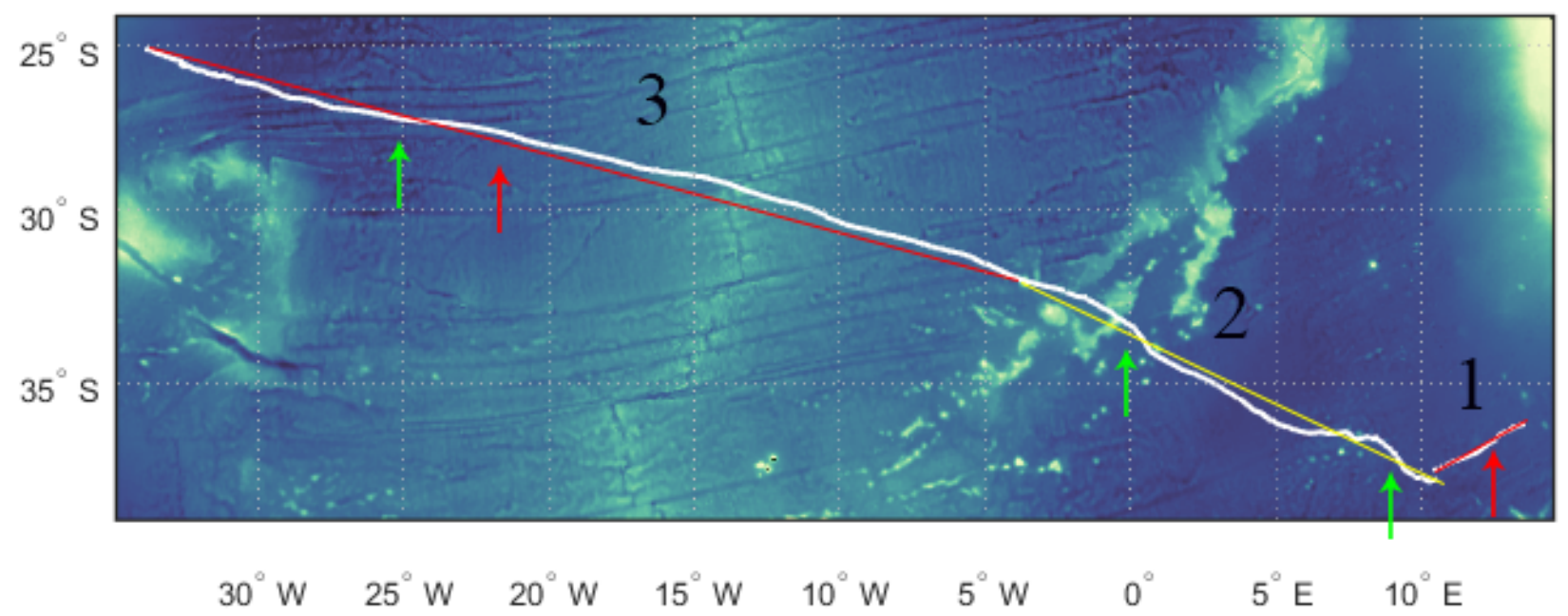

Figure 5. Two regimes of Rossby wave propagation: adhering (red arrows) and overshooting (green arrows). The white line shows the track of the anticyclone crossing the South Atlantic, the red and yellow lines divide track into parts (shown in numbers).

deepest valleys. Our study confirmed the change of angles relative to latitude during eddies cross the ridges (Figure 3). However, the reduction of drift speed during the eddy translation does not exist in many cases (Figure 4).

According to the theory of Korotaev et al. 1997 and Reznik and Dewar 1994 , the trajectories of single eddies at large times propagate asymptotically to the "repose latitude", where it disappears. This type of quasi-parabolic propagation is inherent in very strong Gaussian vortices. The observed longlived eddies of Agulhas are most likely not of this kind. They are weaker and move more smoothly in space. The evolution of such eddies is more likely to fit the vortex model of algebraic spatial fading (see [Reznik and Dewar, 1994, formulas (5.9) and (5.11)]. Analysis of the data from Table 1 is consistent with the results by Reznik and Dewar 1994. Based on Table 1, two main conclusions can be drawn:

- eddy trajectories are rather quasi-linear than quasi-parabolic;

- eddies of smaller spatial sizes are more inclined to move in the meridional direction and have a greater inclination to the parallel.

Analysis of individual tracks gives the following interesting result. At the initial moment, the observed tracks of Agulhas eddies have a distinctly identified tendency towards a certain angle of propagation. The available mathematical models [Early et al., 2011, Korotaev et al., 1997, Reznik, 2010, proceed from the fact that, in general, the motion of eddies is highly nonlinear at large times. However, at the initial stage, at short times, they are linear beta-gyres. Due to the anisotropic dispersion of Rossby waves, long Rossby waves travel to the west, short ones to the east. At the initial stage, the localized axisymmetric formation stretches in the zonal direction, forming the socalled beta-gyres. This is kind of the zero stage of vortex development on the $\beta$-plane. Further, this gyre is already controlled by nonlinearity and leads to a purely meridional displacement, depending on the polarity of the eddy. The principal point of analytical approaches is that linearity is considered unable to give a meridional asymmetry of motion (due to the meridional symmetry of the dispersion relation of linear Rossby waves).

It is believed that linear Rossby waves can explain the East-West asymmetry, but not NorthSouth. However, at the subsequent time stage, it is the nonlinearity that leads to the western displacement of eddies, which is accompanied by the meridional anisotropy. Perhaps this time, when a strictly meridional displacement occurs, is too short, and in Table 1 we are already observing the stage of the so-called adjustment. 
However, it is possible to give another explanation for this fact. The existing analytical models of the dynamics of single vortices on the $\beta$-plane have one significant drawback. Discussing and recognizing certain resonant interactions of a nonlinear vortex and linear Rossby waves emitted or absorbed by it, the authors break the process of vortex initiation and its further evolution. According to Korotaev et al. 1997, "An interesting problem is the entire life cycle of a vortex, starting from its birth due to flow instability or other reasons. Unfortunately, this problem is too complex to be solved now. Instead, we'll focus on the problem of initial values. Although the formation process usually is not considered, the problem of initial values provides a physical understanding of the strong vortex dynamics on the $\beta$-plane". Thus, it is logical to expect that these reasons (of initial values) should play a significant role, at least at the initial stages of eddy evolution. The reasons and methods of eddy generation can be different, but this is not reflected in any way in existing models.

\subsubsection{Evolution of agulhas eddies in the} South Atlantic. We propose the following alternative version of the evolution of the eddy initial stage and do not reject other possible scenarios for mesoscale eddies in other regions. We rely on the statement that eddy dynamics at large times are highly nonlinear. We propose to explain the first stage of eddy nucleation and evolution by the stage of narrowly directed long Rossby wave emission under the combined influence of topography and large-scale non-zonal flow. This can be qualitatively explained as follows.

The Agulhas current, being a strong jet flow, carries with weak captured wave and vortex disturbances, which initially have a small amplitude. In this case, the orbital velocities of the eddies do not differ much from the forward speed, and the nonlinearity parameter (the ratio of the orbital velocity to the forward speed) is approximately equal to one. Colliding with strong topographic changes in the form of a sharp increase in depth, there is a double effect of transforming these disturbances. First, weak eddy formations, which were captured by a strong jet flow, colliding with a sharp increase in depth, due to the conservation law of potential vorticity, strongly compress in diameter, increasing their orbital velocity and, as a consequence, be- come highly nonlinear. Secondly, the current slows down significantly, on the contrary, and it is due to the law of conservation of mass, encountering a sharp increase in depth. Consequently, the nonlinearity parameter sharply increases both due to the numerator, the swirling of the eddies, and due to the denominator, the deceleration of the forward motion of the flow. In this sense, the influence of topography can be characterized as a kind of separator separating the forward motion of the current and eddy formations. Metaphorically speaking, the influence of topography can be described as throwing in pair figure skating. When the partner (current), slowing down, throws out the female partner (eddy), and she greatly increases the rotational movement, then the usual conservation of angular momentum law works. Compressing to the center of her axis of rotation, she (female partner) decreases her moment of inertia but increases the speed of rotation. (The moment of impulse is equal to the product of the speed of rotation and the moment of inertia, and it is constant. When the moment of inertia decreases, the rotation speed increases automatically). In this case, the analogue of the conservation law of the inertia moment is the conservation law of potential vorticity.

The effect described above suggests that at the moment of generation, vortex not only captures the water mass, but also acquires a certain translational and rotational moment, which is a function of the velocity and direction of the large-scale current, as well as topography at a point of the vortex generation, and, as it will be shown below, it also depends on the baroclinic flow parameters in the form of the first baroclinic Rossby radius. This can explain the observed practically rectilinear motion of long-lived eddies of the Agulhas.

Contrary to the model of Korotaev et al. 1997, where the motion of a vortex at the initial stage is determined by some isotropic radiation of Rossby waves, and due to this radiation, the vortex adapts to a hostile environment, we assert the opposite. At the moment of generation, the vortex cannot be coming out of anywhere. The vortex, at the moment of its generation, is a consequence of narrowly directed angular (at an angle to latitude) radiation by the current. At the initial moment, the vortex not only captures a certain volume of water mass but also acquires a certain initial translational and rotational angular momentum, which determines 
all of its further behavior until the moment of disappearance. This is the scenario we are offered by the empirical data obtained in Table 1.

3.3.3. On Rossby waves generated by travelling forcing effects. The importance of the long-wave approximation gives in the linear formulation the following formula for the radiation of Rossby waves by a non-zonal baroclinic flow.

$$
\Psi=\Psi_{0}(x, y)+\varepsilon \varphi_{1}(x, y, z, t), \quad \varepsilon \ll 1,
$$

here $\Psi$ is the quasi-geostrophic stream function, $x, y, z$-axes of a rectangular coordinate system, $t-$ time. Here $v_{1}(x, y)=-\left(\Psi_{0}(y)\right)_{y}^{\prime}$ is a zonal component of the background current velocity, $v_{2}(x, y)=$ $-\left(\Psi_{0}(y)\right)_{x}^{\prime}$ is a meridional component of it. The first term is the background stationary flow, the second term in the equation (1) is linear waves, which can be searched in the form

$$
\varphi_{1}=\varphi_{1}(z) \exp (i(k x+l y-\omega t)),
$$

where $k, l$ are zonal and meridional wavenumbers, $\omega$ is a frequency, $\varphi_{1}(z)$ is a depth-dependent wave amplitude. The effects associated with the nonlinear long-wavelength approximation are outlined in Gnevyshev et al. 2020.

A mathematical model that explains the narrowly directed angular radiation of long Rossby waves by non-zonal currents was formulated by Lighthill 1967. He used the idea to describe the resonance between the flow and the linear divergence-free Rossby waves radiated by the flow. The formula (26) from his paper is

$$
U(k \cos \alpha+l \sin \alpha)\left(k^{2}+l^{2}\right)+\beta k=0,
$$

where $\beta$ is a meridional variation of the Coriolis parameter, and velocity of flow is $\vec{U}=\left(v_{1}, v_{2}\right)=$ $U(\cos \alpha, \sin \alpha)$. If we draw the radiation indicatrix (see Figure 2 of [Lighthill, 1967], corresponding to (2), we get the radiation of waves in a purely westerly direction. This is part of the formation of the $\beta$-gyre, and this leads us again to the statement that it is impossible to obtain the meridional asymmetry in the linear divergence-free problem.

For a better understanding, let us explain the transition from formula (26) in Lighthill's paper (in our article, this is formula (2)) to formula (38) in Lighthill's paper. This formula is the resonance condition for the flow and generated waves. This term is associated with the name of Kelvin, whose ideas were later be developed by Lighthill. Sometimes this formula is called the condition for the stationary of waves in the system associated with their generation source, taking into account the Doppler shift $\Omega=\omega-\vec{k} \vec{U}=0$.

Further, Lighthill assesses how the divergence factor affects the radiation of long Rossby waves. Considering the calculations trivial, he answers immediately in the form of the classical formula (38), which is probably the most physically successful one for representing the widespread idea of the resonance between the motion of the radiator and the waves radiated by it. A similar formula for resonance was used by Kelvin to construct a picture of ship waves [Lord Kelvin, 1906].

Let explain how it turns out. For this, we must modify equation (2) as follows:

$$
U(k \cos \alpha+l \sin \alpha)\left(k^{2}+l^{2}+R_{d}^{-2}\right)+\beta k=0,
$$

here $R_{d}$ is the baroclinic Rossby radius of deformation. In a long-wavelength limit at $k^{2}+l^{2} \ll R_{d}^{-2}$, and taking into account the fact, that the direction of wave radiation (the direction of the normal) at zero frequency will be perpendicular to the quasiplane term near the origin, we obtain a modified formula which is an analogue of formula (38) in the form

$$
\begin{gathered}
\Theta=\arctan \left(\frac{\sin \alpha}{\frac{\beta \cdot R_{d}^{2}}{U}+\cos \alpha}\right)= \\
\arctan \left(\frac{v_{2}}{\beta \cdot R_{d}^{2}+v_{1}}\right),
\end{gathered}
$$

here $\Theta$ is the angle of the direction of wave radiation from the western direction and an angle is measured in the positive sense from the same (westward) direction. The difference between (3) and Lighthill's formula (38) is that Lighthill used a barotropic radius of deformation while we use baroclinic one. If we also used a barotropic radius we would not get any difference from the purely zonal wave direction using this formula. Lighthill concluded that for both nondivergent and divergent Rossby waves, when using the barotropic Rossby radius, the results are practically the same. However, if we use the first baroclinic radius instead of the barotropic one, then the meridional displacement of the waves is observed, and we see a good correspondence of the directions of movement of 
the tracks of the Agulhas eddies in the South Atlantic with the modified Lighthill formula. Perhaps the first baroclinic radius was not yet known in 1967.

The resonance is also mentioned in the model by Korotaev et al. 1997 and Reznik 2010 in a quasi-stationary regime. But it is used there to describe the resonance between a vortex and Rossby waves radiated or absorbed by a vortex. For example, Korotaev et al. 1997 write that a moving vortex on a rotating sphere or the $\beta$-plane excites Rossby waves, just as a moving ship causes ship waves; Rossby waves are observed during the entire period of vortex evolution, but they are very weak for short periods [Reznik, 1992, Reznik and Dewar, 1994. It is easy to see that this condition of linear resonance is in harmony with the nonlinear condition of stationarity if we take the purely zonal case and go from wave motions to localized ones:

$$
p^{2}=\frac{\beta}{v_{1}}+R_{d}^{-2},
$$

here $p$ is a parameter, that sets space scales of the vortex velocity fields can, in principle, depends on time [Reznik, 1992]. Non-zonal currents, regardless of the angle of their propagation, radiate long Rossby waves almost only in the westerly direction. And in this case, we again obtain the classical result of the linear problem, that is, no anisotropy in the north-south direction can be obtained in the linear formulation. However, the fact, that the angle of the radiation of Rossby waves almost does not depend on the angle of direction of the flow, does not physically look quite logical and can only be explained by the long-wave approximation.

Thus, if we use not the barotropic, but the first baroclinic deformation radius $R_{d}$, we get a completely different result. In this case, the spread in values depending on the first baroclinic radius at the place of radiation will be extremely small. It is this fact that we use as the basis for the idea of the radiation of the Agulhas eddies by a nonzonal current, considering the bottom topography and baroclinicity of the current [Gnevyshev and Belonenko, 2020, Gnevyshev et al., 2021. Since the flow velocities at the generation places have approximately the same direction, eddies propagate along the same path (Figure $2 \mathrm{~b}$ ).

Thus, after analyzing the main theoretical approaches [Early et al., 2011. Korotaev et al., 1997 ,
Reznik, 1992, in which the meridional displacement of eddies is discussed, we conclude that no one concept can fully explain this phenomenon since the resulting displacement estimates do not correspond to theoretical formulas. This allows us to conclude that the mechanisms responsible for the meridional displacement of vortices should be different from them.

Based on Lighthill's approach and using his modified formula, we obtain an acceptable comparison of the empirical and theoretical parameters of the meridional displacement of moving eddies: at a $36^{\circ}$ inclination angle of the track, we obtain by formula (3) $\Theta=32-40^{\circ}$, and at a $64^{\circ}$ inclination angle, we obtain at the initial stage of the eddy motion $\Theta=49-58^{\circ}$. We use for the calculations the values of the deformation radius $30-35 \mathrm{~km}$, current velocity $U=5-10 \mathrm{~cm} \mathrm{~s}^{-1}, \beta=1.9 \times 10^{-11} \mathrm{~m}^{-1} \mathrm{~s}^{-1}$. Thus, the observed meridional displacement of eddies in the South Atlantic may be a consequence of the emerging resonance between the non-zonal flow and the wavefield generated by the flow, which manifests in the form of narrowly directed radiation of Rossby waves.

Perhaps the proposed interpretation is not universal for the entire World Ocean, but its application for the South Atlantic has shown its viability.

3.3.4. Effect of the short-wave transformation of Rossby waves near a critical layer. Based on two different approaches (the WKB-approximation and the Cauchy problem), the transformation of Rossby waves on a non-zonal flow is studied [Gnevyshev et al., 2020]. Figure 1a demonstrates that currents in the South Atlantic, where the tracks of eddies are located, are mostly non-zonal. Therefore, the main conclusion of this research is appropriate for the tracks of the Agulhas eddies propagating northwest. Non-zonal flows lead to the splitting of such a concept as a critical layer [Gnevyshev and Shrira, 1989a, 1989b, 1989c, 1989d, 1990. Along with the classical concept of the critical layer, when the waves asymptotically approach slowly the critical layer and the intrinsic frequency is positive, there is another regime of Rossby wave propagation. In the case of non-zonal flow, the wave action (eddy) undergoes a discontinuous jump and the critical layer is crossed twice. Duba et al. 2014, called this regime "overshooting". At first, an intrinsic frequency becomes equal to zero for the finite values of the time and the 
wavenumber components. In this case, the wave action does undergo a discontinuous jump [Duba et al., 2014, and the intrinsic frequency becomes negative which corresponds to Rossby waves of negative energy. Only after that, the reflected wave is approaching again the critical layer asymptotically for a long time. Hence, the effect of the short-wave transformation of Rossby waves near the so-called critical layer exists in two forms: (1) as an adhering, which is a monotonic approaching of wave packets to the critical layer for an infinitely long time. The sign of the intrinsic frequency of the packet remains the same all the time; (2) as an adhering after overshooting when the wave packet, first, crosses its critical layer at finite wavenumber. The wave changes the sign of the intrinsic frequency when overshooting the critical layer and then keeps the sign when it is adhering to this layer asymptotically similar to the previous scenario [Gnevyshev et al., 2020]. The latter regime does not exist for zonal flows. Figure 5 shows examples of these two regimes for the eddies propagating in the South Atlantic.

The finding of this paragraph can be extremely useful and significant in the study of the baroclinicity of Rossby waves. It will lead to a splitting of the spectrum of the vertical Rossby wave problem. Such a scenario has not been previously considered yet some related ideas (multi-level waveguides) have long been known for internal waves (see, for example, [Shrira and Townsend, 2010]. However, the application of these ideas to Rossby waves requires development.

\section{Conclusion}

We analyzed the tracks of long-lived Agulhas eddies, the lifespan of which exceeds 2.5 years, using altimetry data. The main conclusions drawn from the analysis are as follows:

1. Only anticyclones are long-lived eddies of the Agulhas. They have an almost rectilinear motion to the northwest, traveling thousands of kilometers and crossing the South Atlantic.

2. The parameters of eddies (amplitude, radius, orbital velocity, and speed of drift) change significantly during their life. We do not find either an explicit dependence of their displacement on topography or the attenuation of eddies over time. On the contrary, sometimes a seemingly inexplicable short-term increase in the eddy scale occurs (Figure 4, Table 1), which probably is due to the baroclinic factor. This is also supported by the findings of Early et al. 2011, which analyzes the radiation of Rossby waves by a vortex.

3. Table 1 gives estimates of the zonal and meridional displacement of the Agulhas eddies. The zonal displacement and, accordingly, the zonal component of the displacement velocity dominates in comparison to meridional ones, however, in some parts of the track, the vortex displacement to the equator is comparable to their zonal displacement.

4. We have established that the greater the advection velocity of a vortex, the greater its meridional displacement per unit of time.

5. Eddies propagate almost rectilinearly. Under the influence of topography, especially when crossing the ridges, tracks change propagation azimuth, after which they propagate rectilinearly again.

6. The currently existing theories [Early et al., 2011; Korotaev et al., 1997, Reznik, 2010 cannot adequately explain the phenomenon of meridional displacement of Agulhas eddies. In our opinion, the main problem of the inapplicability of most analytical models for interpretation of the meridional displacement is ignoring the initial values. Although the formation process is not considered by us directly, the problem of initial values provides a physical understanding of the strong vortex dynamics on the $\beta$-plane. These reasons should play an essential role, at least at the initial stages of vortex evolution.

7. We propose the alternative theory of the evolution of the Agulhas eddy initial stage and herewith do not reject other possible scenarios for mesoscale eddies in other regions of the World Ocean. We rely on the theory of Lighthill 1967 and, modifying it, explain the meridional displacement of vortices with narrowly directed angular radiation of long Rossby waves by non-zonal currents.

8. The essence of our approach is as follows. We propose to explain the first stage of eddy nucleation and evolution by the stage of narrowly directed long Rossby wave radiation under the combined influence of topography and 
large-scale non-zonal flow. The subsequent dynamics of the vortex are highly nonlinear at large times, yet the law of conservation of potential vorticity is fulfilled. At the moment of generation, the vortex not only captures the water mass but also acquires a certain translational and rotational angular momentum, which are a function of the velocity and direction of the large-scale current, and topography at the point of vortex formation as well. This can explain the almost rectilinear movement of the Agulhas eddies to the northwest.

Data Availability Statement. The authors confirm that the data supporting the findings of this study are available within the article and its supplementary materials.

Acknowledgment. This research was supported by the Russian Foundation of Basic Research, project No 20-05-00066. V.G.G. was supported in the framework of the Shirshov Institute of Oceanology RAS state assignment No 0128-2021-0003.

\section{Electronic Supplement}

One electronic supplement is published below.

1. Supplement $\mathbf{7 7 3 . m p 4}$ (video)

\section{References}

Belonenko, T., V. Zinchenko, et al. (2020), Evaluation of Heat and Salt Transports by Mesoscale Eddies in the Lofoten Basin, Russ. J. Earth Sci., 20, ES6011, Crossref

Benilov, E. S. (2005), Stability of a Two-Layer Quasigeostrophic Vortex over Axisymmetric Localized Topography, Notes and Correspondence p. 123129, Crossref

Biastoch, A., C. W. Boning, J. R. E. Lutjeharms (2008), Agulhas leakage dynamics affects decadal variability in Atlantic overturning circulation, Nature, 456, 489-492, Crossref

Byrne, D. A., A. L. Gordon, W. F. Haxby (1995), Agulhas eddies a synoptic view using Geosat ERM data, J. Phys. Oceanogr., 25, 902-917, Crossref

Chelton, D. B., M. G. Schlax, R. M. Samelson (2011), Global observations of nonlinear mesoscale eddies, Prog. Oceanogr., 91, 167-216, Crossref
Cushman-Roisin, B. (1994), Introduction to Geophysical Fluid Dynamics, Prentice-Hall, Upper Saddle River, N.J.

Duba, C. T., T. B. Doyle, J. F. McKenzie (2014), Rossby wave patterns in zonal and meridional winds, Geophysical \& Astrophysical Fluid Dynamics, 108, No. 3, 237-257, Crossref

Early, J. J., R. M. Samelson, D. B. Chelton (2011), The Evolution and Propagation of Quasigeostrophic Ocean Eddies, J. Phys. Oceanogr., 41, 1535-1555, Crossref

Giulivi, C. F., A. L. Gordon (2006), Isopycnal displacements within the Cape Basin thermocline as revealed by the hydrographic data archive, Deep Sea Res. Part I., 53, 1285-1300, Crossref

Gnevyshev, V. G., T. V. Belonenko (2020), The Rossby paradox and its solution, Hydrometeorology and Ecology. Proceedings of the Russian State Hydrometeorological University, 61, 480-493, (in Russian $\longdiv { \text { Crossref } }$

Gnevyshev, V. G., V. I. Shrira (1989a), Dynamics of Rossby wave packets in the vicinity of the zonal critical layer taking into account viscosity, Izv. Akad. Nauk SSSR, Fiz Atmos. Okeana, 25, No. 10, 10641074.

Gnevyshev, V. G., V. I. Shrira (1989b), Kinematics of Rossby waves on non-uniform meridional current, Okeanologiya, 29, No. 4, 543-548.

Gnevyshev, V. G., V. I. Shrira (1989c), On the evaluation of barotropic-baroclinic instability parameters of the zonal flows in beta-plane, Dokl. Akad. Nauk. SSSR, 306, No. 2, 305-309.

Gnevyshev, V. G., V. I. Shrira (1989d), Transformation of monochromatic Rossby waves in the critical layer of the zonal current, Izv. Akad. Nauk SSSR, Fiz. Atmos. Okeana, 25, No. 8, 852-862.

Gnevyshev, V. G., V. I. Shrira (1990), On the evaluation of barotropic-baroclinic instability parameters of zonal flows on a beta-plane, J. Fluid. Mech., 221, 161-181, Crossref

Gnevyshev, V. G., S. I. Badulin, T. V. Belonenko (2020), Rossby waves on non-zonal currents: structural stability of critical layer effects, Pure Appl. Geophys., Crossref

Gnevyshev, V. G., A. V. Frolova, et al. (2019), Interaction of Rossby waves with a jet stream: basic equations and their verification for the Antarctic circumpolar current, Izvestiya, Atmospheric and Oceanic Physics, 55, No. 5, 412-422,

Gnevyshev, V. G., A. V. Frolova, et al. (2021), Topographic Effect for Rossby Waves on a Zonal Shear Flow, Fundamentalnaya $i$ Prikladnaya Gidrofizika, 14, No. 1, 4-14, Crossref

Gordon, A. L., W. F. Haxby (1990), Agulhas eddies invade the South Atlantic: Evidence from Geosat altimeter and shipboard conductivity-temperaturedepth survey, J. Geophys. Res. C: Oceans, 95, 3117-3125, Crossref

Guerra, L. A. A., A. M. Paiva, E. P. Chassignet (2018), On the translation of Agulhas rings to 
the western South Atlantic Ocean, Deep-Sea Research Part I, 139, 104-113, Crossref

Kamenkovich, V. M., Y. P. Leonov, et al. (1996), On the Influence of Bottom Topography on the Agulhas Eddy, Journal of Physical Oceanography, 26, No. 6, 892-912, Crossref

Korotaev, G. K., A. B. Fedotov (1994), Dynamics of an isolated barotropic eddy on a beta-plane, J. Fluid Mech., 264, Crossref

Korotaev, G. K., V. L. Dorofeev, A. B. Fedotov (1997), Dynamics of an intensive isolated barotropic eddy in the presence of background vorticity, Physical Oceanography, 8, 1, Crossref

Leonov, Y. P. (1995), Vliyaniye topografii na vikhri Aguliasskogo techeniya, Avtoreferat dissertatsii kand. fiz.-mat. nauk, Institut Okeanologii im. P. P. Shirshova, Moscow. (in Russian)

Lighthill, M. J. (1967), On waves generated in dispersive systems by travelling forcing effects, with applications to the dynamics of rotating fluids, Journal of Fluid Mechanics, 27, No. 4, 725, Crossref

Lord Kelvin (1906), Deep Sea Ship-Waves, Proc. $R$. Soc. Edinburgh, 25, 1060, Crossref

Malysheva, A. A., A. A. Kubryakov, et al. (2020a), Agulhas Leakage Estimation Using Altimetry and Argo Data, Issledovanie Zemli iz Kosmosa, 2, 2434. (in Russian)

Malysheva, A. A., A. A. Kubryakov, et al. (2020b), Estimating Agulhas Leakage by Means of Satellite Altimetry and Argo Data, Izvestiya, Atmospheric and Oceanic Physics, 56, 1581-1589, Crossref

Morrow, R., F. Birol, et al. (2004), Divergent pathways of cyclonic and anti-cyclonic ocean eddies, Geophys. Res. Lett., 31, L24311, Crossref

Nezlin, M. V. (1986), Rossby solitons (Experimental investigations and laboratory model of natural vortices of the Jovian Great Red Spot type), Sov. Phys. Usp., 29, 807-849, Crossref
Reznik, G. M. (1992), Dynamics of singular vortices on a beta-plane, Journal of Fluid Mechanics, 240, 405-432, Crossref

Reznik, G. M. (2010), Dynamics of localized vortices on the beta plane, Izvestiya. Atmospheric and Oceanic Physics, 46, No. 6, 784-797, (in Russian Crossref

Reznik, G. M., W. K. Dewar (1994), An analytical theory of distributed axisymmetric barotropic vortices on the $\beta$-plane, Journal of Fluid Mechanics, 269, 301-321, Crossref

Reznik, G. M., Z. Kizner (2010), Singular vortices in regular flows, Theoretical and Computational Fluid Dynamics, 24, No. 1-4, 65-75, Crossref

Reznik, G. M., T. B. Tsybaneva (1994), The effect of topography and stratification on planetary waves in the ocean, Okeanologiya, 34, No. 1, $5 . \quad$ (in Russian)

Shrira, V. I., W. A. Townsend (2010), Inertiagravity waves beyond the inertial latitude. Part 1. Inviscid singular focusing, J. Fluid Mech., 664, 478509, Crossref

Travkin, V. S., T. V. Belonenko (2019), Seasonal variability of mesoscale eddies of the Lofoten Basin using satellite and model data, Russ. J. Earth Sci., 19, No. 5, ES5004, Crossref

Williams, S., M. Petersen, et al. (2011), Adaptive extraction and quantification of geophysical vortices, IEEE Transactions On Visualization and Computer Graphics, 17, No. 12, 2088-2095, Crossref

Corresponding author:

T. V. Belonenko, Saint Petersburg State University, 7-9, Universitetskaya nab., Saint Petersburg 199034, Russia.(btvlisab@yandex.ru) 